\section{P180 AETIOLOGY OF BRONCHIECTASIS IN A NON-SPECIALIST SERVICE. HOW DOES COPD AFFECT THE PICTURE?}

doi:10.1136/thoraxjnl-2012-202678.241

A Crabtree, EH Baker. St George's, University of London, London, UK

Background In tertiary care, bronchiectasis (BE) patients are a highly selected group, often with a complex aetiology or associated disease. The national British Thoracic Society bronchiectasis audit provided us with an opportunity to characterise our secondary care bronchiectasis population. We hypothesised that the majority of bronchiectasis seen in our non-specialist service would be associated with chronic obstructive pulmonary disease (COPD). We therefore determined the prevalence of $\mathrm{COPD} / \mathrm{BE}$ and compared investigation and management of $\mathrm{COPD} / \mathrm{BE}$ patients to those with bronchiectasis alone.

Methods Patients with a clinical diagnosis of bronchiectasis were identified in respiratory outpatients between $1^{\text {st }}$ October and $30^{\text {th }}$ November 2011 during the national bronchiectasis audit period. Those with bronchiectasis confirmed on CT thorax were included. Data including aetiology, lung function and other investigations were retrieved from electronic records.

Results Forty seven patients were identified, age $65 \pm 17$ years (range 19 to 88 years), 57\% female. Forty percent (19) had COPD Other common aetiologies were post-infection (26\%), asthma (21\%) and idiopathic (11\%). The table compares characteristics between bronchiectasis patients with and without COPD. COPD/BE patients were older and had a significantly greater pack year smoking history. Similar investigations were performed in the two groups. COPD/BE patients had significantly worse airflow obstruction and gas transfer than bronchiectasis patients without COPD COPD/BE patients were more likely to have Pseudomonas aeruginosa, Methicillin Resistant Staphylococcus aureus or coliforms cultured from sputum, but antibiotic treatment and prophylaxis was similar in the two groups. Other medications were similar in the two groups, although COPD/ $\mathrm{BE}$ patients were more likely than those without COPD to be prescribed long acting anti-cholinergic medication.

Conclusion In our secondary care setting, COPD is the most common underlying condition associated with bronchiectasis. COPD/ $\mathrm{BE}$ patients share many clinical characteristics with other bronchiectasis patients and have worse lung function and more pathogenic microorganisms isolated from sputum culture. The emerging association between COPD and bronchiectasis requires further research to improve the characterisation and management of this patient group.

\section{P181 ADHERENCE TO TREATMENT IN PATIENTS WITH BRONCHIECTASIS INFECTED WITH PSEUDOMONAS AERUGINOSA}

doi:10.1136/thoraxjnl-2012-202678.242

${ }^{1} \mathrm{~A}$ R McCullough, ' $\mathrm{CM}$ Hughes, 'M Tunney, ${ }^{2} \mathrm{JS}$ Elborn, ${ }^{1} \mathrm{JM}$ Bradley. ${ }^{1}$ Clinical \& Practise Research Group, School of Pharmacy, Queen's University Belfast, Belfast, Northern Ireland; ${ }^{2}$ Centre for Infection Immunity, Queen's University Belfast, Belfast, Northern Ireland

Introduction Patients with bronchiectasis and $P$. aeruginosa infection are often prescribed a complex regimen of inhaled antibiotics, other respiratory medicines and airway clearance techniques (ACT). Current bronchiectasis guidelines recognise that adherence to treatment is important in this population but no published studies have determined the level of adherence to treatment in patients with bronchiectasis infected with $P$. aeruginosa.

Aim The aim of this study was to determine adherence rates to inhaled antibiotics, other respiratory medicines and ACT in patients with bronchiectasis infected with $P$. aeruginosa.

Methods Patients with bronchiectasis (confirmed by HRCT) were recruited from hospital respiratory clinics if they had a positive sputum culture for $P$. aeruginosa and were using inhaled antibiotics.
Prescription refill data were used to calculate percentage adherence by dividing the amount collected by the amount prescribed, multiplied by 100 . Participants were categorised as adherent (score $\geq 80 \%$ ) and non-adherent (score $<80 \%$ ) to inhaled antibiotics and other respiratory medicines using this method. Participants completed the modified Self-reported Medication-taking Scale for ACT (score $0-5$; adherent score $\geq 4$, non-adherent score $<4$ ). Spirometry was performed according to ATS/ERS guidelines. Chi square tests were used for between group analyses.

Results 75 participants were recruited: 24M/51F; mean (SD) age 64 (8) yrs; FEV 61 (25) \% predicted. Sixty-four (85\%) participants were prescribed colomycin, $11(15 \%)$ were prescribed tobramycin, 68 (91\%) were prescribed bronchodilators and 65 (87\%) were prescribed inhaled corticosteroids. All participants were prescribed ACT; active cycle of breathing techniques $(n=39,53 \%)$ and Acapella $^{\circledR}(n=45,61 \%)$ were most commonly prescribed. Eleven percent $(16 \%)$ participants were adherent to all treatments. Fifty-two percent and $51 \%$ of participants were adherent to inhaled antibiotics and other respiratory medicines, respectively. Thirty-nine percent of participants were adherent to ACT. Adherence category varied significantly between inhaled antibiotics and other respiratory medicines $(p=0.04)$, with $34 \%$ of participants being adherent to one treatment and not the other.

Conclusion Patients with bronchiectasis patients infected with $P$. aeruginosa have a high burden of treatment. Only $11 \%$ of patients were adherent to all treatments, half were adherent to medicines and even fewer were adherent to ACT, indicating that patients make decisions about which treatments to use.

\section{P182 UNDERLYING CAUSES OF BRONCHIECTASIS IDENTIFIED IN A SPECIALIST NON-CF BRONCHIECTASIS SERVICE}

doi:10.1136/thoraxjnl-2012-202678.243

${ }^{1} \mathrm{KE}$ Leighton, 'RJ Stretton, ${ }^{2} \mathrm{E}$ Furrie, ${ }^{2} \mathrm{~S}$ Marshall, 'TC Fardon, 'S Schembri, ${ }^{3}$ JD Chalmers. 'Department of Respiratory Medicine, Ninewells Hospital, Dundee, United Kingdom; '2Department of Immunology, Ninewells Hospital, Dundee, UK; 3University of Dundee, Dundee, UK

Introduction Identifying the cause of non-CF bronchiectasis can have important implications for future treatment. The British Thoracic Society (BTS) issued guidance for testing in bronchiectasis in 2010 but many of these recommendations are based on expert opinion only (grade D evidence). We describe the underlying causes identified using the BTS recommended testing regime at a specialist non-CF bronchiectasis service.

Methods The study included patients attending a tertiary bronchiectasis clinic over 1 year (April 2011-April 2012). The diagnosis of bronchiectasis was made by high resolution CT. Sputum microbiology for the previous 2 years was used to determine colonisation status. A respiratory physician and immunologist assigned the underlying cause after discussion, following standardised testing recommended by the BTS guidelines.

Results 88 patients had CT confirmed bronchiectasis. The median age was 66 years (Interquartile range 57-73). 39 patients (44.3\%) were male. The median number of lobes involved on CT was 2 (IOR 1-3). 51 patients were classified as idiopathic. 14 patients had ABPA, 8 patients had connective tissue disease, 4 patients were classified as post-infective, 3 patients had inflammatory bowel disease, 3 patients had an identified immunodeficiency, 2 were classified as secondary to COPD, 1 patient had chronic reflux, 1 patient had a congenital malformation and 1 patient had Mounier-Kuhn syndrome.

In the idiopathic cohort, 19 patients were colonised with Haemophilus influenzae, 7 with Streptococcus pneumoniae, 7 with Pseudomonas aeruginosa, 4 with Moraxella catarrhalis, 3 with Staphylococcus aureus and 5 patients with enteric gramme negative organisms. The remainder were not colonised. 